\title{
Which User of technology? Perspectivising the UTAUT model by application of the SFL language Pronoun System towards a systems perspective of technology acceptance and use
}

\author{
Cheryl Marie Cordeiro*
}

Centre for International Business Studies (CIBS), School of Business, Economics and Law, University of Gothenburg, 405 30, Sweden

\author{
A R T I C L E IN F O \\ Article history: \\ Received: 24 February, 2018 \\ Accepted: 09 April, 2018 \\ Online: 25 April, 2018
}

Keywords:

Research methodology

Unified theory of acceptance and

use of Technology (UTAUT)

Systemic functional linguistics

(SFL)

International business

Technology management

Remote service

\begin{abstract}
A B S T R A C T
This study applies systemic functional linguistics (SFL) as complementary framework of analysis of technology acceptance models (TAMs). The purpose is to bridge research methodology language in international business (IB) studies and engineering management science. Currently TAMs and its consolidated version, the Unified Theory of Acceptance and Use of Technology (UTAUT) provides for a typology of one user in one context scenario. The need for the UTAUT model to account for multiple users in multiple work contexts in a single framework of analysis was foregrounded in the study of the workflow processes of a remote services business model of a European founded multinational business enterprise (MBE) with regards to its (i) intra-firm improvements in managing remote services cases, and its (ii) extra-firm selling of life cycle management remote services contracts. The Enterprise has global operations in over 100 countries, of which this study focused on its European operations of improving the quality of remote services for the marine industry. Through an application of SFL unto UTAUT, this study illustrates how multiple users in multiple contexts can be analysed simultaneously, and whose behaviours can be accounted for in a single framework of analysis. The combined SFL UTAUT model addresses the initial statisticity of the UTAUT model, whilst at the same time, expands upon current theoretical perspectives of technology use and acceptance that can be applied in practice.
\end{abstract}

\section{Introduction}

Technology acceptance models (TAMs) [1-3] and its consolidated version, the Unified Theory of Acceptance and Use of Technology (UTAUT) [4,5] whilst widely applied to various industry context [6-8], currently provides for a typology of a single user to a single context of use of a specific technology [912]. In an era of converging technologies and digital platforms across multiple work spaces, a less static, more sophisticated approach towards the study of technology use and acceptance is needed.

The purpose of this study is to bridge research methodology in language in international business (IB) and engineering management science. It applies the pronoun system found in systemic functional linguistics (SFL) $[13,14]$ from language science as complementary framework of analysis to the UTAUT

${ }^{*}$ Corresponding Author: Cheryl Marie Cordeiro, University of Gothenburg, Sweden. Email: cheryl.cordeiro@handels.gu.se model. Most technology acceptance studies are quantitative oriented studies. This study contributes to the existing literature by extending the UTAUT model applications by use of SFL, a primarily qualitative analysis approach. The effect of applying the pronoun system in SFL unto UTAUT enables for a simultaneous analysis of multiple users in multiple context of use for a single technology. The need for a multiple user, multiple context perspective in the study of technology use and acceptance arose in the context of studying the workflow processes of a remote services business model of a European founded multinational business enterprise (MBE) with regards to its (i) intra-firm improvements in managing remote services cases and its (ii) extra-firm securing of remote services life cycle management contracts. The Enterprise of study has global operations in over 100 countries, of which this study focuses mostly on its European operations of improving the quality of remote services for the marine industry. The simultaneous understanding of intra- and extra- firm UTAUT processes was necessary for a systems 
integral view of the overall Enterprise remote services business model.

A systems perspective $[15,16]$ of the remote services business model for the Enterprise was needed, because if there was a bottleneck of product-services efficiency, the Enterprise should be able to identify the area of inefficiency quickly. In order to do this, a systemic systems overview of workflow operations is necessary by top management of the Enterprise. This called for a research practice method that could give a systems integral perspective of UTAUT. But the simultaneous application of the UTAUT model posed a challenge in different contexts in its current form due to the linear staticity of perspective i.e. one UTAUT model for one context, targeted to understand one type of pre-defined User. The aim of this research methods study is to illustrate how this linear staticity can be circumvented by the application of the pronoun system in systemic functional linguistics (SFL) as theory and framework of data analysis. This is done by collecting and analysing data from both intra-firm and extra-firm perspectives given by Enterprise and Enterprise-related respondents for remote services technology use and acceptance.

This paper begins with a literature review of UTAUT and SFL as fields of research, focusing on UTAUT and SFL as methods for research. This study contains two methods sections. The first pertains to the Enterprise remote services marine sector as case example in illustration of how the SFL pronoun system can be applied to the UTAUT model to unfold various User perspectives. The second methods section illustrates how SFL can be combined in complementarity to the UTAUT model, to form an integrated SFL UTAUT model in studying technology acceptance and use. This is followed by a findings and discussion section, where the empirical findings are presented incorporating a discussion on the SFL UTAUT combined model. This paper ends with an outline of study limitations, future research directions and conclusions.

\section{Literature Review}

\subsection{Technology Acceptance Studies}

The past forty years have seen scholars design theories and models in understanding of the influencing elements of acceptance and use of technologies. During the 1970s, the Theory of Reasoned Action (TRA) was put forth by Fishbein and Ajzen [1], which explained a person's behavioural tendencies with the aim of predicting changes and interpreting particular personal behaviour. TRA was formulated based on the assumption that behaviour is shaped by intentions that in turn depend on personal attitudes and subjective norms. A decade later, the Theory of Planned Behaviour (TPB), was proposed as an extension of TRA, working on the assumption that behaviours could be controlled by certain parameters in context [2]. In similar timeframe, Davis, Bagozzi and Warshaw [3] proposed the Technology Acceptance Model (TAM) to explain the causal relationships between internal psychological variables such as beliefs, attitudes and behavioural intention and actual information technology (IT) system. The widely studied and considered valid TAM model was based on the two constructs of the User, which was Perceived Usefulness (U) and Perceived Ease of Use (E). These constructs were considered effective when applied to the understanding (even predicting) of individual acceptance behaviour across various information technologies and their users. Subsequent models developed include the combined TAM and TPB that focused on the impact of experience of the use of technology [4], the TAM2/TAM 3 models as a theoretical extension of the TAM that included the perspectives of subjective norms and job relevance when accepting the use of new technologies [5,6], and the Unified Theory of Acceptance and Use of Technology, or UTAUT [13].

UTAUT [6] arose from a comprehensive conceptual review and empirical study of eight technology acceptance models. The unified model consists of six broad constructs deemed to be significant direct determinants of technology acceptance and use that include:

(i) performance expectancy (PE) - the degree to which an individual believes that using the system will help them improve on job performance

(ii) effort expectancy (EE) - the degree of ease of use by the individual of the system

(iii) social influence (SI) - the degree to which the individual perceives it important that others perceive them to use the new system

(iv) behavioural intention (BI) - the degree to which the individual intends to use the system

(v) use behaviour (UB) - degree of affect on the part of the individual when using the system

(vi) facilitating conditions (FC) - the degree to which the individual feels they have the resources and support (technical / organizational) to use the system

An additional four constructs that moderate technology acceptance and use are gender, age, experience and voluntariness of use. Figure 1 illustrates UTAUT as it appears in Venkatesh et al. [13:447]. UTAUT continues to be widely used across various technology management studies even if other models of technology acceptance such as the Model of Acceptance with peer support [14] and the Content Acceptance model [15] have been proposed.

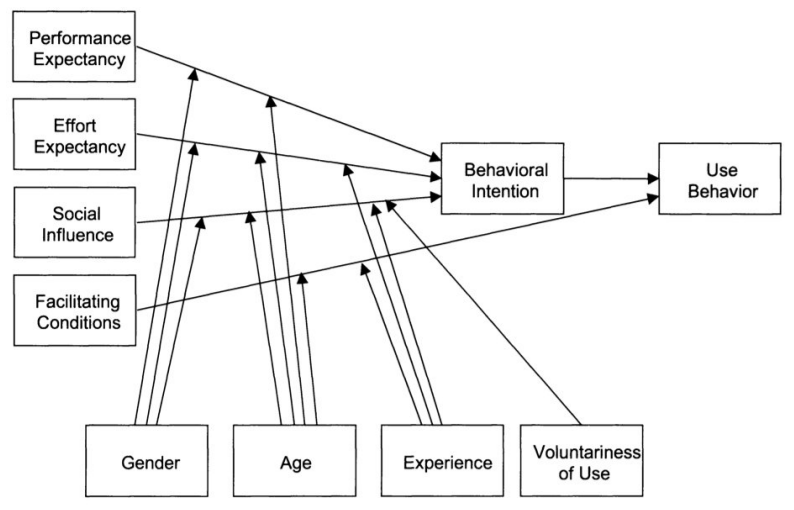

Figure 1. Model of Unified Theory of Acceptance and Use of Technology (UTAUT) [13: 447].

The UTAUT model generally focuses on the causal (cause and effect) relationship between individual attitudes towards using a technology, personal tendencies towards using a technology, actual use of a technology and identifying performance expectancy of a technology. In this model, FCs are taken as the main determinant factor in the use of a technology or system [6].

But while the model of technology acceptance predominantly explain a User's behavioural expectation [16] and intention to use 
[13], perceived ease of use [17,18] and actual use [6,7] in context (facilitating conditions), most studies have implicitly defined the User in a linear model of product-service to the User. In this construct, the User is often defined as the business enterprise endcustomer/user that includes a broad range of social actors that include customers [8,19,20], teachers / students [21-24], academics, physicians and nurses [25-27], civilians and military personnel $[28,29]$.

The linear product-service workflow from enterprise to customer as (end-)User disregards within enterprises users who range from service technicians to engineers and product lifecycle managers. This internally defined enterprise users of technology are those who work in support of or are even the creators of the technology for enterprise end-customer/user. The enterprise internal users need to use the same technologies or technological platform to support the product-service workflow from enterprise to end-customer/user. The linear UTAUT concept flow from Engineer to Customer use poses a challenge to the model's inability to overlay contexts of use from intra- to extra- enterprise environments, leaving the perspective of Engineer to Engineer as a field of knowledge under researched.

\subsection{Systemic Functional Linguistics}

Language is an open adaptive system of choice that humans use language to describe and circumscribe reality [30-32]. We use language in its functional purpose both as process of acting/transacting through time, and as product that helps us situate and identify ourselves in relation to others [33-35]. "Functional linguistic theories are predicated on the claim that language is first and foremost a means for communication between human beings, and that this fact has a deep and allpervasive influence on the forms that languages take." [36:619]. Language is a resource for making meaning and meaning resides in systemic patterns of options so that language presents itself as a system network potential for meaning $[9,10,40]$.

The usefulness of the UTAUT model when used in research design and method is that it condenses the semiotics (meaning) attributed by Users to technology used in context. Applying UTAUT helps researchers find answers to why and how Users accept and use technology. User experiences and interactions with technology are expressed through the language system. Because the language system is open and adaptive, the factors abstracted and condensed into UTAUT more often condenses and delimits the full experiential context expressed by Users. In a complementarity, systemic functional linguistics (SFL) is a theory and model of language as a social semiotic system. SFL as theory allows us to account for how language enables us to communicate with each other and with our surrounding in the manner that we do [36]. In that sense, language is its own meta-language, because the language system can be used to study language typology, as well as subjects of other disciplines i.e. other systems. In order to simultaneously apply UTAUT in different contexts of use, this study turns to the underlying unifying theory and framework of language, reflected in the SFL theory and model of language.

The architecture of language reflected in SFL comprises sets of systems and options. When people talk, what is said is usually derived from systemic choice, and SFL gives an open adaptive framework to a general theory of meaning. Language in use is a "system network [that] can represent any domain of activity where choosing can be analysed into small closed sets of options. This does not imply that all such sets of options will be independent of each other - in language they never are." [30:20]. To that end, it would be the consistent dialogic of text and talk carried out by individuals, between individuals and groups of individuals that would also have the power to influence, circumscribe and shape the intersectional relations between producers and users of technology product-services.

Digitalization and the context of Industry 4.0 creates an environment of converging technologies that increases interconnectivity. This increased connectivity between humans and between humans and technology, is also reflected in parallel with the meaning creating system in language when systems simultaneous (rather than dependent) functioning increases its semiotic potential [37]. As such, in the case of the application of UTAUT in understanding technology acceptance, what is needed is a more holistic perspective of not just how a set of defined Users use and accept technology, but how the technology in itself is shaped by feedback from Users in a process of co-evolution of technology product-services. SFL is used in this study to show how it can provide a meta-language framework that allows for the investigation of these dialogic processes between Producers and Users of technology in a context that form today's system of modern ecological habitus. As Bourdieu's [38 81ff] notion of habitus has it, "logogenesis provides the material (i.e. semiotic goods) for ontogenesis, which in turn provides the material for phylogenesis; in other words, texts provide the means through which individuals interact to learn the system" and it is through this individual heteroglossic aggregation that a social system evolves. What needs to be investigated in such an interconnected habitus is simultaneity in UTAUT application. This is the ability and capacity for research design and methodology to perspectivise systematically, all actors in all contexts, so that one part of the system can be studied in relation to other parts of the system, larger or smaller. This study focuses in particular, the pronoun system of language that perspectivises deictic points of view such as $I$ (You), We (They), It and Its applied to the simultaneous application of UTAUT in different contexts. The research questions addressed are:

RQ1: How can SFL be applied to the UTAUT model towards simultaneous use to reflect intra- and extra- firm perspectives of User acceptance and use of technologies?

RQ2: What contributing factors can be established by using SFL as language theory and framework to broaden the applicability of UTAUT into various contexts of use?

\section{Method: Case Example}

\subsection{Remote Services in the Marine Sector as Enterprise Case Example}

The business model of the Enterprise of study since its founding in the late $1800 \mathrm{~s}$ is sales of products from manufacturing in a business to business context. In the past twenty years, its business model has needed to shift from manufactured products alone to digitally connected product-services. The operations of the Enterprise spans over 100 countries with about 135000 employees working in global teams that speak different languages 
across different business sectors. In the era of digitalisation and the Industry Internet of Things (IIoT), the Enterprise began to face differing national and regional data sharing policies as potential barriers to efficiently providing customers with advanced product-service business solutions. Empirical data for this study comes in the form of semi-structured interviews and shadowing of engineers from field studies doing remote services and product maintenance in the marine sector. The field studies were conducted in the Enterprise's office locations for the marine sector. These offices were located in 5 European countries that include Finland, Netherlands, Norway, Sweden and Switzerland (regional remote service centre headquarters location).

Remote services in the Enterprise case example context is defined as a type of system maintenance that allows for a roundthe-clock remote monitoring and observation of Enterprise manufactured products. It is part of the Enterprise's advanced services portfolio for the marine sector. Built on big data analytics, such systems of maintenance potentially allow for longer upkeep times of automation processes, sending out alarm signals to Enterprise system engineers, reporting potential system failures and predicting hardware life spans so that components can be changed prior to breakdown. In its efforts towards providing advanced services to its customers, the Enterprise is currently in the phase of building shared digital platforms, both intra- and extra- Enterprise.

The concurrent building of software platforms internal and external to the Enterprise in address of Enterprise efficiency, gave rise to a synchronous duo-User of technology scenario with an overlapping work process timeframe. The first scenario (ScenA) is of a need for a shared internal remote services platform for improved work efficiency for Enterprise employees. ScenA will help employees better coordinate their work efforts across departments, across local business units (LBUs) and its European regional remote services centre located in Switzerland. The second scenario (ScenB), is of a shared external platform for customers that interfaces with ScenA. ScenB is so that the Enterprise remote services team can have continuous contact with its global clients when the vessels are out at sea. ScenA and ScenB Users comprise this duo-User scenario.

\subsection{Respondent Profile}

In terms of the UTAUT model, what was needed was thus a congruent, synchronous understanding of User acceptance and use of technologies for both ScenA and ScenB, for which the User is differently defined. ScenA would have User defined as Enterprise employees. ScenB would have User defined as Enterprise end-customer, purchasers of the Enterprise remote service systems. Both sets of defined Users for ScenA and ScenB are not homogenous groups of individuals because ScenA for example consists of different employee profiles from Enterprise top managers, system engineers to customer service personnel. ScenA User profiles might also be regionally dispersed, working in different Enterprise business units from headquarters to local business units (LBUs). With most sea faring vessels regionally unconfined, ScenB User profile is also variegated to customers who are located in different parts of the world and who speak different languages. It is not unusual for ScenB Users to encounter different vessel product system use and regional policies. ScenB respondent feedback for this study comes the Enterprise's Product Life Cycle Managers who act as points of contact between the Enterprise and its end-customers. Enterprise end-customers can be located as far as Singapore in Southeast-Asia and are referred to the closest remote services centre in times of distress signals or need of maintenance.

Although the Enterprise has different departments managing remote services that could in total include more than 400 employees in different job functions, there was a challenge in setting up field studies that coincided with respondent time availability for interviews and shadow operations. Enterprise Engineers for example, had to be onsite for both interviews and shadowing of operations to be conducted. As such the total number of respondents available for representation in this study was limited in relation to the given research project timeframe. Interviews and focus group discussions were held with the following Enterprise individuals that categorised as ScenA Users:

- 9 Remote Services Engineer (Headquarters and Local Business Units). These individuals are located at global Enterprise headquarters (Switzerland) that also serves as regional remote service centre for the marine sector. Engineers have different types of specialist knowledge and expertise levels, ranging from Engineering Level 1 (for expert knowledge) and Engineering Level 2 and 3 for lower expertise levels and experience. Cases that cannot be solved for the end-customers are escalated in accordance to specialist knowledge from LBUs to the global centre. Engineers are on call for end-customers 24/7, with no allowance for more than 2 hours downtime for the endcustomer. Some engineers are also building the remote diagnostics and maintenance platform whilst the Enterprise is providing this as product-service to the end-customers, so that the engineers in this aspect are both Producer and User of the (same) technology platform.

- 3 Field Engineers. These individuals are located both at global headquarters and at LBUs. Depending on area of expertise and knowledge, the field engineer closest to the vessel is deployed. They are on call $24 / 7$, and can be flown (helicopter or private plane) to vessel sight with immediate notification. Some field engineers in the Enterprise have moved on to the role of Product Life Cycle Managers.

- 2 Marine Remote Service Customer Service (RSCS) Personnel. These individuals are usually the first point of contact between Enterprise end-customers the remote services engineers. They might also receive and manage calls for Enterprise related questions, not pertaining to remote services. Most calls from Enterprise end-customers for remote services in the marine sector are time critical. The remote services team have a response time of maximum two hours to get the parts/components onsite to the vessels. The challenge for these respondents is to get the correct connection between Enterprise expert engineer for its endcustomer. They are Users of the Enterprise internal general computer services platform. Calls are almost always initiated by the Enterprise end-customers.

- 2 Enterprise top managers (Headquarters). Top management of the Enterprise consist of a team of individuals in leadership position, although all technological decisions have to be 
approved by the Enterprise Chief Technology Officer (CTO) regardless of Enterprise division. The respondents here are important as decision makers and in their capacity to steer technology strategies for the Enterprise for long-term sustainable business. Their main interest is to develop a future internal standardised Enterprise interface that connects the departments of various divisions (even beyond the marine sector). This internal interface should have a corresponding standardized external interface for when end-customers login to their accounts. As such, some aspects of remote services need to have a consistent Enterprise branding and theme that is both employee and end-customer user friendly. A consistent brand and theme for digital interfaces for the Enterprise has been challenging to achieve due to the relative autonomity of LBU workings, and different workgroups within the Enterprise.

Enterprise respondents categorised for ScenB Users include:

- 6 Enterprise Product Life Cycle Managers (LCM). These individuals often have advanced engineering degrees and some have worked as field engineers prior to becoming LCMs. Their role is different from the RSCS personnel in the sense that the LCMs take care of end-customer needs from product purchase to product life-end or recycle of components. Part of their job is to sell diagnostics and service maintenance contracts. Acting as key account managers, they are in long-term direct feedback contact with Enterprise marine sector end-customers $(\mathrm{EeC})$. Their feedback for this study is assumed reliable with regards to feedback from endcustomers for the following reasons - (i) LCMs are in constant contact with EeCs due to an earnest effort in improving product-services portfolio for future technology developments, and towards future Enterprise sustainable business competitiveness and (ii) LCMs are held responsible by EeCs for product-service downtime, a poor follow-up of which might affect future service contract agreements.

Respondents were targeted specifically for product-service expert knowledge and because of experience and exposure to both intra- and extra- systems use that was to be improved upon by the Enterprise. All respondents are involved in providing advanced services in the Enterprise in different capacities and areas of expertise, the number of years spent with the Enterprise ranged from 2 to 24 years. Those who have spent 2 years at the Enterprise had mostly joined as master thesis students, who had then gained expert knowledge in a specific field or product before being fully employed by the equal opportunities employer Enterprise.

\section{Method: SFL Pronoun System Combined With Constructs of UTAUT}

The pronoun system of language in use, which is $I$ (You), We (They), It and Its, serves as a reference point system to indicate point of view in answer to the questions of who is acting / saying and what about, under which circumstance. It is a language referencing perspectivity system that can be mapped in a four quadrant model (Figure 2). All pronoun perspectives in their various forms, singular/plural, subjective/intersubjective, objective/interobjective, can encompass an "inner" and "outer" experience and can be expressed as such. This model, reflecting of the evolving nature of language, is inherently adaptive and relative in perspective, depending upon researcher definition of the unit of analysis of what is singular/plural, subjective/objective etc. Consistent inquiries from each perspective will render a specific type of knowledge that can be classified under the eight primordial methodological perspectives such as phenomenology (singular subjective), hermeneutics, ethnomethodology, autopoiesis, empiricism and systems theory (plural interobjective). As such, the four quadrants can be said to reflect a type of knowledge related to that perspective of inquiry, reflecting both inner and outer worldviews.

Figure 2 maps the different perspectives. Within the SFL system, the semiosis and expression of inner and outer human experiences are reflected through transitivity processes in the metafunction of language, amongst them are acts of doing (material processes), acts of saying (verbal processes), or thinking (mental processes), indicated through use of verbs. Each transitivity processes encompasses agents who act within an activity context and circumstance.

In the Upper Left (UL) quadrant is the singular subjective perspective of 'I'. When applying UTAUT to technology, the assumed User would have this Agency / Actorship of 'I' reflected in the UL quadrant, such as "I use this technology for $x$ purposes" or "Using this technology helps me accomplish <task $x>$ ". Collected knowledge of UTAUT constructs related to User's expectancy of use can be reflected in the UL quadrant. But the perspective is relative, depending on who is the defined 'I'. In ScenA, the 'I' User would be the Enterprise engineers and remote customer services personnel. In ScenB, it would be the Enterprise's end-customer who uses the remote services produced by the Enterprise. The Lower Left (LL) quadrant reflects the plural intersubjective perspective of "We". This collective perspective tends to reflect an ideology of shared perspectives attributed to the proximity of working with others in the same environment, given allowance for slight variation of these shared experiences. Knowledge collected on UTAUT constructs pertaining to social influence (corporate culture) or voluntariness of use (intra- inter-group workings and social norms) are reflected in the LL quadrant.

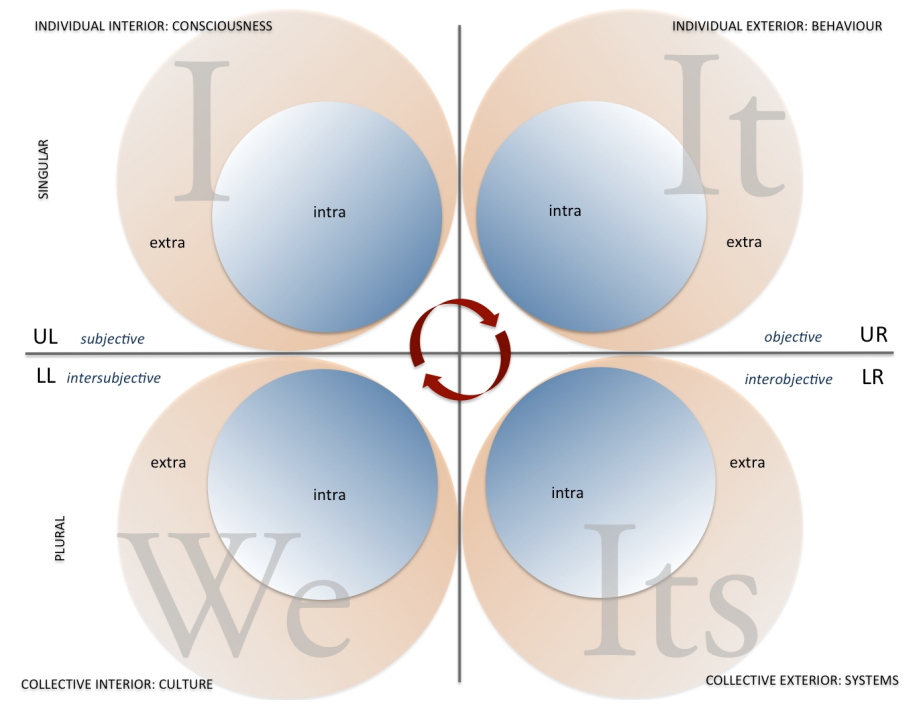

Figure 2. The pronoun system of language in use, mapped in four quadrants (adapted from Wilber [39]). 
The Upper Right (UR) and Lower Right (LR) quadrants reflect singular objective and plural interobjective perspectives. Knowledge collected on UTUAT constructs pertaining to a particular technology 'It' and the technology system, 'Its', to which a particular technology belongs are reflected in these quadrants. In this study, it is noted that ScenA presents a perspective from the Enterprise engineers who design and implement the technology and technology system, to which they are Users of these systems themselves. ScenB presents a perspective of the Enterprise end-customer as User who purchases the technology and technology system from the Enterprise. Their technology acceptance experience is different, but both types of Users will need to feedback their experiences of use of technology into the Enterprise system in order for improvement in efficiency of Enterprise remote services delivery.

The perspectives, beginning in the UL quadrant working anticlockwise presents perspectives that increase in complexity. The perspectives move from Individual to Group to Object/s in a system to a Systems view reflected in the LR quadrant. The LR quadrant that reflects a systems view of the subject of inquiry presents the broadest perspective, one that encompasses all other perspectives. All perspectives are in dialogic relation with each other, where a change in one perspective could well influence the context of the other perspectives. The broadest perspective reflected in the LR quadrant encompasses all other perspectives.

The SFL pronoun system model is useful for the simultaneous application of UTAUT in different contexts of use because the four quadrants unfold in a systemic manner, not just the Agency of a material/verbal action but it uncovers (human) Agency in relation to the object of use (UTAUT's type of technology) and circumstance of use (UTAUT's facilitating conditions).

\section{Findings and Discussion}

Figure 3 shows constructs of UTAUT for ScenA mapped into the SFL pronoun system quadrant, where Enterprise engineers as defined Users. Figure 4 shows constructs of UTAUT for ScenB mapped into the SFL pronoun system quadrant, where Enterprise end-customers are defined Users. The insights for ScenB Users come from Enterprise Product Life Cycle Managers who are in direct contact with the customers. All elements found in the quadrant perspectives are interrelated and in dialogic relationship to each other. The dialogic relations between the Actors are signalled by arrows drawn in circularity. All actions take place through the context of spacetime. Time is indicated as a fundamental background feature in which Enterprise activities and processes take place.

\subsection{ScenA UTAUT SFL Pronoun System Findings}

Due to that this study is specific in its Users for ScenA being Enterprise top managers, engineers and remote services customer service personnel, the normative definition of User often studied by application of the UTAUT model is slightly modified to reflect the different roles as Users of a single platform system. The single platform system is one that encompasses the marine sector remote diagnostics and product maintenance system both built and used by Enterprise engineers and LCMs. What is different for the different Users of this system is the Time perspective. Enterprise top managers have a longer timeline perspective, in view that one
Enterprise technology strategy is to build a standard computer software platform for internal and external use. This standard platform of computer supported services in remote services necessarily interfaces with those used by the Enterprise endcustomers, for them to independently login and check on product diagnostics. The Enterprise engineers have different roles, some have project timelines as short as 3 months to come up with computer support. A binding factor for ScenA Users is shared organizational values in technological innovation and service excellence towards end-customers.

ScenA Users hold various expert capacities who worked with the building of the Enterprise products and services that were to be sold to ScenB Users. As such, UTAUT moderating constructs [13] such as 'social influence', 'gender', 'age' and 'voluntariness of use' could have potentially played secondary influencing roles in this study to other factors such as 'experience' that ranked high on the Users list. Most Users for ScenA were expert knowledge workers and the 'social influence' could be redefined as the Enterprise culture of technological innovation, and pioneering remote services work not just in the marine sector but in other heavy industries in which the Enterprise has business operations. UTAUT models also predict User behaviour based on cognitive state of the user, their expectation and intention. These factors for ScenA users were subsumed under a broader corporate culture context of quality excellent in the product-services produced towards end-customers. This then needed a shift in perspective for the application of the UTAUT model towards a broader unit of analysis than the individual as User but towards the Enterprise top management defined as 'User' in this context.

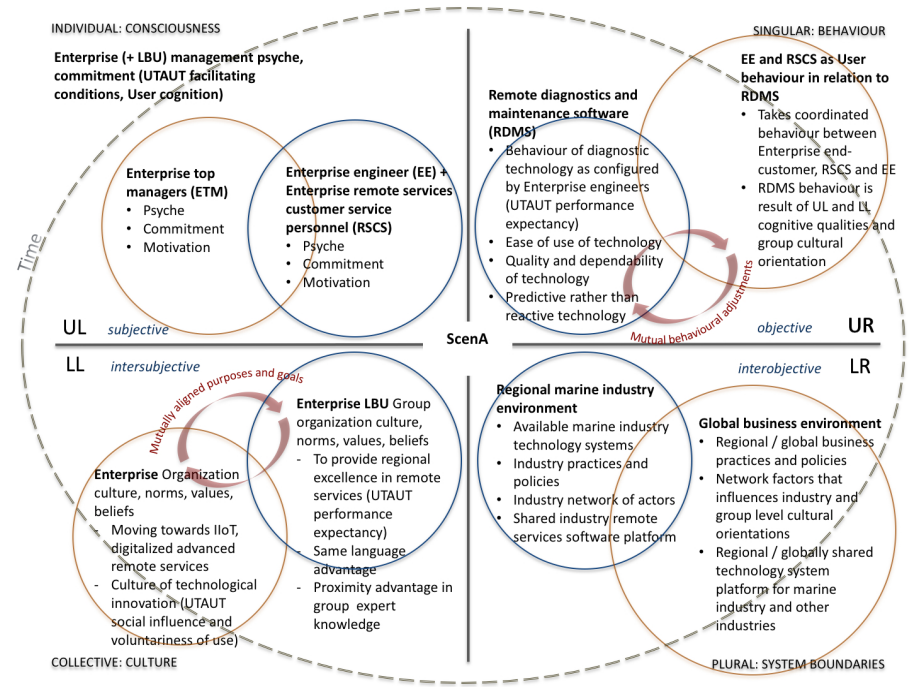

Figure 3. SFL pronoun system perspective of UTAUT Users for ScenA: Users from the Enterprise

The Enterprise top management defined as 'User' towards new technologies comes in the form of Enterprise psyche, commitment and motivation (Figure 3, UL quadrant) of first producing the technology and then using it themselves, as platform towards an integrated advanced services portfolio in an era of digitalisation. To some extent Users of technology will need to be convinced of their intention to use that technology even prior to first testing. In the case of ScenA Users, the respondent feedback from field studies and interviews indicated that the Enterprise engineers 
would not (and could not) produce the remote diagnostics and maintenance platform if top management at the Enterprise were not (financially) commitment and believed that this technology would contribute to their competitiveness and to their endcustomer's industry competitiveness. It is here that the SFL pronoun system is effective in helping researchers perspectivise and define 'which User' of technology, giving the research model more fluidity and adaptiveness for research purposes, so that the psyche and commitment of the top management of the Enterprise can be accounted for in understanding technology acceptance and use from the Enterprise perspective.

The Enterprise top management commitment reflected in the UL quadrant in Figure 3 not only bolsters both employee motivation and commitment at individual level but it encourages the building of a corporate culture towards constant technology innovation, reflected in the LL quadrant in Figure 3. Enterprise top management discourse would filter through the organization through management hierarchies targeted at group level meetings where Enterprise engineers and remote service customer service personnel would be motivated to provide excellent remote services for their end-customers. This organizational culture of technological innovation creates an understanding towards the building of an Enterprise internal common remote services platform in order to connect LBU employees with its global and regional centres for remote services. The main challenge experienced here, reflected in the LL quadrant in Figure 3, is the communication between the LBUs located in different European countries, and the Enterprise remote services headquarters in Switzerland. Two main challenges arose on why it was difficult building a common internal remote services platform that include (i) preference for local language and (ii) existing remote services technology platform that did not correlate with the architecture of the updated platform as suggested by headquarters.

\subsection{ScenB UTAUT SFL Pronoun System Findings}

Feedback from Enterprise end-customers (EeC Users, Figure 4) come from Enterprise LCMs who have the role of key account managers, who secure and follow-up on remote services life cycle management contracts with end-customers. LCM interview responses are assumed earnest due to that the proper managing of accurate end-customer feedback is crucial in building future remote services support for both Enterprise and end-customer use. The long-term Enterprise technology strategy is to have a shared platform of remote services between Enterprise and its endcustomers. A challenging task much due to that currently, most end-customers are geographic proximity bound by registration of country of ownership of vessel, even if the vessels are globally seafaring. Different types of software systems are used, how much data information the systems can share between themselves are regulated by industry, national and regional policies. The challenge for seafaring vessels and remote services is that vessels lack consistent internet connections due to inconsistent satellite connections. As such, for varying reasons, Enterprise endcustomers tend to be contextually (geographic proximity) bound in their immediate business networks when it comes to remote diagnostics and maintenance. Should a sea vessel come into crisis, it is referred to the nearest regional remote services centre or an LBU. Enterprise LBUs, although belonging to the Enterprise, work sometimes in competition with other to secure end- customers. LBUs have the advantage of local language, proximity to end-customers and shared remote services platform systems with their end-customers, built from more than twenty years ago. The strength of the LBU's system is also the weakness of the Enterprise's system due to that LBUs tend to have separate tools and systems to track cases that are not shared with the Enterprise global headquarters. This contributes to the challenges faced by ScenA Users whose current technology strategy is to create a common working platform for Enterprise and its EeCs. While there are Enterprise European initiatives to coordinate between departments and LBUs located in Europe to create a single platform for services, at the time of study, this has yet to be achieved. As such, Enterprise end-customer feedback is crucial and timely information is needed by the LCM in order to work towards a common shared platform for remote services.

The $\mathrm{LCM} / \mathrm{EeC}$ as User perspective is reflected in Figure 4. The interdependent relations between Enterprise and EeCs are reflected in the UL and LL quadrants in Figure 4, where both Users of remote services need to share a sense of commitment to using the system bolstered by a general movement towards digitalisation and the Industry Internet of Things (IIoT). EeC corporate culture will also need to be one that is progressive and welcoming to technology innovation and change. Some motivating indicators as to why $\mathrm{EeC}$ would want to use Enterprise provided remote diagnostic and maintenance service and why they would want a signed contract would be SFL UTAUT constructs pertaining to the UR quadrant of singular objective 'It'. Reflected also in the summary findings in Table 1, what $\mathrm{EeC}$ Users find critically important is that the remote services provided is quick (time critical), reliable, user friendly systems that require little effort in learning from the $\mathrm{EeC}$ User. This is not to say that EeCs do not wish to put in learning time for acquired Enterprise systems. Rather, that the learning time should be short. EeCs also have as corporate goal, to be independent users of acquired systems, so that they can fix problems themselves without needing to go back to the Enterprise LCMs or call Enterprise crisis lines.

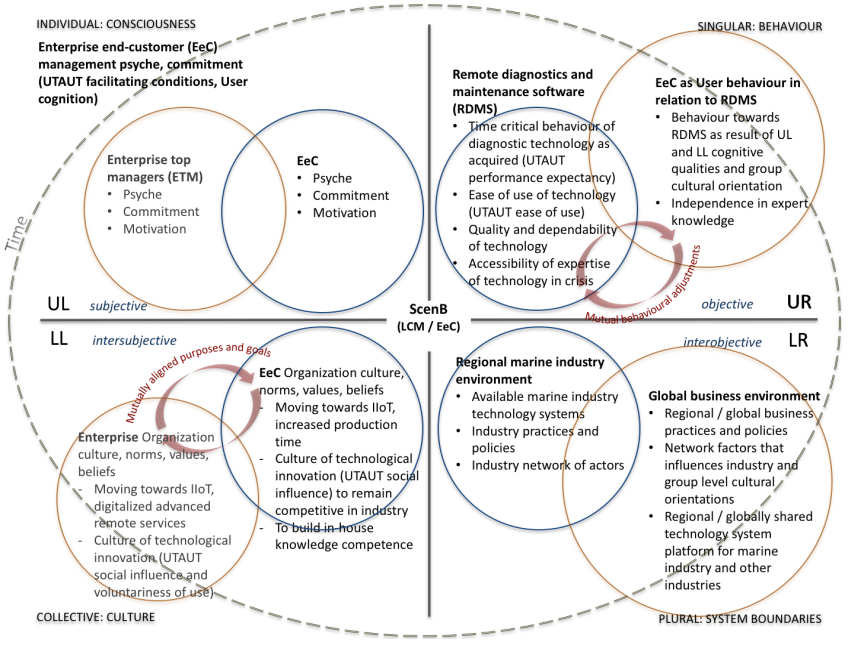

Figure 4. SFL pronoun system perspective of UTAUT Users for ScenB: Enterprise end-customer

The desire to be independent is due to $\mathrm{EeC}$ corporate sensitive and private data/information where it is not appreciated if Enterprise engineers can login remotely on unsecured internet connections to access $\mathrm{EeC}$ corporate systems data. EeCs are not 
welcoming of what they consider to be an intrusion of privacy. How can EeC trust that it is an Enterprise engineer and not a competitor who has logged in was one such question raised to an Enterprise LCM, even if confidentiality agreements have been signed. EeCs also tend to want to keep operating costs down, so that having a remote diagnostics and maintenance contract is deemed useful when the system is predictive rather than reactive in feedback. This allows for action to be taken prior to failure. A movement towards reuse and recycling of parts also lowers costs for EeC. But even as EeCs might be aware of what more advanced remote services might offer, the incompatibility with older, existing systems takes time to disinvest or incorporate with newer systems and services.

Table 1. SFL UTAUT constructs for technology acceptance for combined Users: Enterprise, Enterprise engineers and Enterprise end-customers

\begin{tabular}{|c|c|c|c|c|c|}
\hline SFL defined Users -> & $\begin{array}{c}\text { ETM } \\
\text { (ScenA) }\end{array}$ & $\begin{array}{l}\text { EEHQ } \\
\text { (ScenA) } \\
\end{array}$ & $\begin{array}{l}\text { EELBU } \\
\text { (ScenA) }\end{array}$ & $\begin{array}{c}\text { RSCS } \\
\text { (ScenA) }\end{array}$ & $\begin{array}{c}\begin{array}{l}\text { LCM/EeC } \\
\text { (ScenB) }\end{array} \\
\end{array}$ \\
\hline \multicolumn{6}{|l|}{$\begin{array}{l}\text { SFL UTAUT } \\
\text { constructs }\end{array}$} \\
\hline $\begin{array}{l}\text { Performance expectancy } \\
\text { (singular subjective, 'I') }\end{array}$ & - & $\mathrm{c} v$ & $\mathrm{c} v$ & $\sqrt{ }$ & - \\
\hline $\begin{array}{l}\text { Performance expectancy } \\
\text { (plural intersubjective, } \\
\text { 'We') }\end{array}$ & $\sqrt{ }$ & $\sqrt{ }$ & $\sqrt{ }$ & $\sqrt{ }$ & - \\
\hline $\begin{array}{l}\text { Performance expectancy } \\
\text { (singular objective, 'It') }\end{array}$ & $\sqrt{ }$ & $\mathrm{c} v$ & $\mathrm{c} v$ & $\mathrm{c} v$ & $\mathrm{c} v$ \\
\hline $\begin{array}{l}\text { Performance expectancy } \\
\text { (plural interobjective, } \\
\text { 'Its') }\end{array}$ & $\mathrm{fV}$ & $\mathrm{f} V$ & - & - & $\mathrm{f} v$ \\
\hline $\begin{array}{l}\text { Effort expectancy } \\
\text { (singular subjective, 'I') }\end{array}$ & - & $\checkmark$ & $\sqrt{ }$ & $\checkmark$ & $\sqrt{ }$ \\
\hline $\begin{array}{l}\text { Effort expectancy } \\
\text { (plural intersubjective, } \\
\text { '(We') }\end{array}$ & $\sqrt{ }$ & $\sqrt{ }$ & $\sqrt{ }$ & $\sqrt{ }$ & $\sqrt{ }$ \\
\hline $\begin{array}{l}\text { Effort expectancy } \\
\text { (singular objective, 'It') }\end{array}$ & $\bar{c} \sqrt{ }$ & $\bar{c} \sqrt{ }$ & 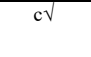 & $\begin{array}{cl}\sqrt{ } \\
\text {. }\end{array}$ & 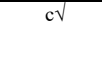 \\
\hline $\begin{array}{l}\text { Effort expectancy } \\
\text { (plural interobjective, } \\
\text { 'Its') }\end{array}$ & $\mathrm{fv}$ & $\mathrm{fv}$ & - & - & $\mathrm{fv}$ \\
\hline $\begin{array}{l}\text { Social influence } \\
\text { (singular subjective, 'I') }\end{array}$ & - & - & - & - & - \\
\hline $\begin{array}{l}\text { Social influence } \\
\text { (plural intersubjective, } \\
\text { 'We') }\end{array}$ & $\checkmark$ & $\sqrt{ }$ & $\sqrt{ }$ & $\checkmark$ & $\checkmark$ \\
\hline $\begin{array}{l}\text { Social influence } \\
\text { (singular objective, 'It') }\end{array}$ & $\mathrm{c} \sqrt{ }$ & $\mathrm{c} \sqrt{ }$ & $\sqrt{ }$ & $\sqrt{ }$ & $\mathrm{c} v$ \\
\hline $\begin{array}{l}\begin{array}{l}\text { Social influence } \\
\text { (plural interobjective, } \\
\text { 'Its') }\end{array} \\
\end{array}$ & $\mathrm{fv}$ & $\mathrm{fv}$ & $\sqrt{ }$ & $\sqrt{ }$ & $\mathrm{fV}$ \\
\hline $\begin{array}{l}\text { Behavioural intention } \\
\text { (singular subjective, 'I') }\end{array}$ & $\sqrt{ }$ & $\sqrt{ }$ & $\sqrt{ }$ & $\sqrt{ }$ & $\sqrt{ }$ \\
\hline $\begin{array}{l}\text { Behavioural intention } \\
\text { (plural intersubjective, } \\
\text { 'We') }\end{array}$ & $\mathrm{c} \sqrt{ }$ & 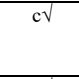 & $\sqrt{ }$ & $\sqrt{ }$ & $\mathrm{c} v$ \\
\hline $\begin{array}{l}\text { Behavioural intention } \\
\text { (singular objective, ' 'It') }\end{array}$ & $\mathrm{c} v$ & $\mathrm{c} v$ & $\mathrm{c} v$ & $\mathrm{cl}$ & $\mathrm{c} v$ \\
\hline $\begin{array}{l}\text { Behavioural intention } \\
\text { (plural interobjective, } \\
\text { 'Its') }\end{array}$ & $\mathrm{f} v$ & $\mathrm{fv}$ & $\sqrt{ }$ & $\sqrt{ }$ & $\mathrm{f} \nu$ \\
\hline $\begin{array}{l}\text { Use behaviour } \\
\text { (singular subjective, 'I') }\end{array}$ & - & - & - & - & - \\
\hline $\begin{array}{l}\text { Use behaviour } \\
\text { (plural intersubjective, } \\
\text { 'We') }\end{array}$ & - & - & - & - & - \\
\hline $\begin{array}{l}\text { Use behaviour } \\
\text { (singular objective, 'It') }\end{array}$ & $\bar{c} \sqrt{ }$ & $\mathrm{c} \sqrt{ }$ & 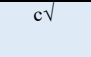 & $\mathrm{cl}$ & $\mathrm{c} \mathrm{d}$ \\
\hline $\begin{array}{l}\text { Use behaviour } \\
\text { (plural interobjective, } \\
\text { 'Its') }\end{array}$ & $\mathrm{f} v$ & $\mathrm{fv}$ & $\mathrm{fv}$ & $\mathrm{fv}$ & $\mathrm{fV}$ \\
\hline $\begin{array}{l}\text { Facilitating conditions } \\
\text { (singular subjective, 'I') }\end{array}$ & $\mathrm{c} v$ & $\mathrm{c} \sqrt{ }$ & $\mathrm{c} v$ & $\mathrm{c} v$ & $\mathrm{c} v$ \\
\hline $\begin{array}{l}\text { Facilitating conditions } \\
\text { (plural intersubjective, } \\
\text { 'We') }\end{array}$ & $\mathrm{c} v$ & $\mathrm{c} \sqrt{ }$ & $\mathrm{c} v$ & $\mathrm{c} v$ & $\mathrm{c} v$ \\
\hline $\begin{array}{l}\text { Facilitating conditions } \\
\text { (singular objective, 'It') }\end{array}$ & $\mathrm{c} v$ & $\mathrm{c} v$ & $\mathrm{c} v$ & $\mathrm{c} v$ & $\mathrm{c} \sqrt{ }$ \\
\hline $\begin{array}{l}\text { Facilitating conditions } \\
\text { (plural interobjective, } \\
\text { 'Its') }\end{array}$ & $\mathrm{fV}$ & $f v$ & $\mathrm{f} V$ & $\mathrm{fV}$ & $\mathrm{f} V$ \\
\hline
\end{tabular}

'Its')

ETM $=$ Enterprise top management, $\mathrm{EEHQ}=$ Enterprise engineers, Headquarters

EELBU $=$ Enterprise engineers, Local Business Unit,

RSCS $=$ Remote services customer service personnel

$\mathrm{LCM} / \mathrm{EeC}=$ Product Life Cycle Managers (reflecting Enterprise end-customers)

\subsection{Combined SFL UTAUT Users Findings Summary}

Table 1 summarises the findings of the SFL pronoun system defined Users, in relation to the six broad UTAUT category constructs for technology acceptance when placed into the SFL pronoun system quadrants.

Factors such as 'gender', 'age', 'experience' and 'voluntariness of use' are incorporated into the more specific pronoun system quadrants. The factors of 'gender', 'age' and 'experience' were accounted for in respondent profile through the primary requirement that all respondents were knowledge experts in their field regardless of gender or age. The Enterprise emphasises it is an equal opportunity employer. The combined Users include perspectives from Enterprise top managers, Enterprise engineers, remote services customer service personnel and Enterprise end-customers who feedback directly to Enterprise Product LCMs. Table 1 indicates which technology acceptance constructs are relevant (by ' $\sqrt{ }$ ), which are critically relevant (by ' $\mathrm{c} \sqrt{ }$ ') and those that will be relevant in future (by ' $\mathrm{f} \sqrt{ }$ '). UTAUT constructs with an indeterminate answer or that are non-relevant are indicated by '-'.

In overview, Table 1 indicates that what was defined as 'relevant', 'critically relevant' and 'future relevant' as UTAUT construct, depended upon the User perspective, as helped defined by the SFL pronoun system. In keeping with organization area of expertise, that the Enterprise defines itself as world leader in innovative technology, what was deemed as critically relevant for all Users was SFL UTAUT construct Effort expectancy (singular objective, 'It'), which meant that the remote services technology was expected to work for both the Enterprise and its end-customer. The technology was produced to be end-customer user friendly and accessible, so that learning input from the end-customer, whilst necessary, would be kept at minimal level. Critically relevant was UTAUT facilitating conditions for all SFL pronoun system perspectives as Users. This could be due to the fact that without adequate motivation from Individual level to conducive environment at Systems level, the technology would not be able to survive or evolve in the human technological ecosystem. Facilitating conditions also had to be right if a future (thus future relevant, ' $\mathrm{f} V$ ') remote services standard platform for regional and global use is to be built / implemented. Its development will depend upon Enterprise commitment as well as customer demand and supporting industry policies and practices.

\section{Study Limitations and Future Research}

The strengths of this study are also its limits. This includes (i) using the Enterprise as case example unit of analysis and Enterprise only respondents, and (ii) using the SFL pronoun system in offering of a plurality of perspectives to UTAUT User constructs. Pertaining to strength and limitation (i), Enterprise as case example provides this study with a cohesive organization environment in which research method theory and analysis can be done. What has been assumed are shared corporate cultures and shared corporate strategies. The single organization as case example might in that sense crave comparative studies to be made, perhaps by definition of industry sector, even across industry sectors, comparing remote services technologies. Still, while single organization shared values has shown to be mostly true, it was not true that the Enterprise and its LBUs were homogeneous 
in their workflows. This might have proved to be a challenge with regards to placing all Enterprise respondents into the majority ScenA Users. This is because it reflects that ScenA Users share the same User experience, when in effect, there are variations of context of use for ScenA system Users, which are not fully accounted for in this study due to that the focus of this study was to illustrate how SFL could be used to unfold different versions of Users usually statically defined when applying UTUAT models. The capturing of User nuances in remote services could in this case, be a subject area for future research, in view that remote services would be a field that grows in application with increased digitalisation of industry processes, beyond the marine sector into robotics and process automation. Since language is still its own metalanguage, SFL could be a means of a cohesive theory and framework towards a general systems theory perspective of studies on technology innovations and combined product-services. There were practical challenges to obtaining data for this study that included time coordination for respondents to be present during the field study and for shadowing purposes.

Pertaining to strength and limitation (ii), the use and application of the SFL pronoun system, while offering an unfolding of perspectives on any subject of study, is also reliant on clear definition of research purpose and design on the part of the researcher. Relativity of perspective $x$, will need to be defined, in relation to point of interest $y$, in context $[n+\ldots]$ in order to be effective in use. While the SFL pronoun system framework opened up various User definitions for UTAUT and made troubleshooting easier, in the sense that the SFL UTAUT defined User could be exactly pinpointed for unease of use of technology for example, what still remained were the working processes of communication between Enterprise business units and their endcustomer on how to improve remote services efficiency. It could also be said that the SFL pronoun quadrant approach might not be too appreciated with a management or practitioner audience because the fluidity of perspectives as exacting as it might be, is also confusing, depending on audience. The remote services customer service personnel for example, who were the individuals meeting crisis calls from end-customers had little concern for how much commitment top management had towards building a standardised internal remote services platform for the future. They were rather more concerned that they could pass on the crisis call to an immediate available remote services engineer.

\section{Conclusion}

The purpose of this study is to bridge research methodology across different disciplines. In particular, it is to apply a language science model of analysis the SFL, unto a model of technology use and acceptance, UTAUT. This was done because a practical need arose to address multiple users of technologies in multiple work contexts in a single framework of analysis. The need for a plurality of perspectives of defined User (i.e. which user of technology?) arose in a case example of an Enterprise needing to develop a shared remote services platform for employees (mostly engineers) as well as end-customers. In such a context, both Enterprise engineers and Enterprise end-customer were Users of this technology system. UTAUT in its original application, is a model of technology use and acceptance that renders a linear statically defined User to a single context of use. Because language is inherently human and we use language to express and encode many internal and external experiences and world views, this study turned to SFL as functional language theory and framework of data analysis with the questions of (RQ1) how SFL can be applied to the UTAUT model so that the UTAUT model can be simultaneously applied for differently defined Users in different contexts and (RQ2) what contributing factors can be established by use of SFL as language theory and framework that broadens the applicability of UTAUT into various contexts? To that end, in answer to RQ1, this study has illustrated how the architecture of language contains in itself a myriad of language systems, of which the pronoun system is one. The SFL pronoun system unfolds primordial perspectives of Agency and Actorship set in both group and environmental context. In answer to RQ2, this unfolding of perspectives by applying the SFL pronoun system is what allows for the plurality of views of the defined Users of UTAUT constructs. The plurality of User views in different contexts when combined with UTAUT constructs allows for the immediate identification of disjunctive views occurring in the business workflows, when an Enterprise engineer believes the system to be user friendly but when the Enterprise end-customer does not. Or when two Enterprise engineers have different User experiences of the same system. This SFL UTAUT model allows for such gap in knowledge identification, and it gives those who work in the context a chance to reconcile these differences in opinion and work towards closing the knowledge gaps. The fluidity of the SFL pronoun system that also maps different types of knowledge zones also means that researchers can use the pronoun quadrant model to visualise research design perspective and address gaps in knowledge.

The application of the SFL pronoun system in complement to the UTAUT model is novel in research methodology. The resulting combined SFL UTAUT theoretical construct and the subject of study of remote services could be better developed by similar type multi-enterprise studies or comparative multienterprise type studies. And perhaps what the SFL UTAUT construct does not and cannot do, is to address the actual communication patterns between Actors and their surrounding context. Miscommunication is identifiable by the SFL UTAUT construct, but the act of improving on communication across different Enterprise business units and between their endcustomers remains very much a human cognitive process.

\section{Conflict of Interest}

The author declares no conflict of interest.

\section{Acknowledgment}

This study is funded by Stiftelsen Riksbankens Jubileumsfond of Sweden, as part of its Flexit (2015-18) Program, RMP15-0954:2. The purpose of the main project is to investigate linguistic methodological applications of analysis to user acceptance and use of technology.

\section{References}

[1] Fishbein, M., Ajzen, I., Belief, attitude, intention and behavior: An introduction to theory and research, Reading, MA: Addison-Wesley Publishing Company, 1975

[2] Ajzen, I., "The theory of planned behaviour", Organ Behav Hum Dec, 50(2), 179- 211, 1991. http://dx.doi.org/10.1016/0749-5978(91)90020-T 


\section{C.M. Cordeiro et al. / Advances in Science, Technology and Engineering Systems Journal Vol. 3, No. 2, 309-318 (2018)}

[3] Davis, F. D., Bagozzi, R. P., Warshaw, P. R. "User acceptance of computer technology: A comparison of two theoretical models", Manage Sci, 35, $982-$ 1003, 1989. http://www.jstor.org.ezproxy.ub.gu.se/stable/2632151

[4] Venkatesh, V., Bala, H., "Technology acceptance model 3 and a research agenda on interventions", Decision Sci 39(2), 425-478, 2008. https://doi.org/10.1111/j.1540-5915.2008.00192.x

[5] Taylor, S., Todd, P.A. “Assessing IT usage: The role of prior experience", MIS Quart 19(2), 561-570, 1995. http://www.jstor.org/stable/249633

[6] Blackwell, C., Lauricella, A., Wartella, E., Robb, M., Schomburg, R., "Adoption and use of technology in early education: The interplay of extrinsic barriers and teacher attitudes: The interplay of extrinsic barriers and teacher attitudes", Comput Educ, 69, 310-319, 2013. https://doi.org/10.1016/j.compedu.2013.07.024

[7] Haas, S., Senjo, S., "Perceptions of effectiveness and the actual use of technology-based methods of instruction: A study of California criminal justice and crime-related faculty", J Crim Just Educ, 15(2), 263-285, 2004. https://doi-org.ezproxy.ub.gu.se/10.1080/10511250400085981

[8] Thakur, R., "Customer adoption of mobile payment services by professionals across two cities in India: An empirical study using modified technology acceptance model”, Bus Perspect Res 1(2), 17-30, 2013. https://doi-org.ezproxy.ub.gu.se/10.1177/2278533720130203

[9] Venkatesh, V., Morris, M.G., Davis, G.B., Davis, F.D., "User acceptance of information technology: Toward a unified view", MIS Quart 27(3), 425-478, 2003. http://www.jstor.org.ezproxy.ub.gu.se/stable/30036540

[10] Barelka, A., Jeyaraj, A., Walinski, R., "Content acceptance model and new media technologies", J Comput Inform Syst 53(3), 56-64, 2013. https://doiorg.ezproxy.ub.gu.se/10.1080/08874417.2013.11645632

[11] Sykes, T.A., Venkatesh, V., Gosain, S., "Model of acceptance with peer support: A social network perspective to understand employees' system use", MIS Quart 33(2), 371-393, 2009. http://www.jstor.org.ezproxy.ub.gu.se/stable/20650296

[12] Maruping, L., Bala, H., Venkatesh, V., Brown, S., "Going beyond intention: Integrating behavioral expectation into the unified theory of acceptance and use of technology", J Assoc Inf Sci Technol 68(3), 623-637, 2017. http://dx.doi.org/10.1002/asi.23699

[13] Halliday, M.A.K., Matthiessen, C., Halliday's Introduction to functional grammar, $4^{\text {th }}$ ed. Abingdon: Routledge, 2014

[14] Halliday, M.A.K., Meaning as choice. In, L. Fontaine, T. Bartlett and G. O'Grady (eds.), Systemic functional linguistics: Exploring choice, Cambridge University Press, p. 15-26, 2013.

[15] Capra, F., \& Luisi, P. (2014). The Systems View of Life: A Unifying Vision.

[16] Capra, F. (2005). Complexity and Life. Theory, Culture \& Society, 22(5), 33-44

[17] Martins, C., Oliveira, T., Popovič, A., "Understanding the Internet banking adoption: A unified theory of acceptance and use of technology and perceived risk application", Int J Inform Manage, 34(1), 1-13, 2014. https://doi.org/10.1016/j.ijinfomgt.2013.06.002

[18] Saadé, R. G., Kira, G., "Mediating the impact of technology usage on perceived ease of use by anxiety", Comput Educ, 49(4), 1189-1204, 2007. https://doi.org/10.1016/j.compedu.2006.01.009

[19] Shin, D., "Determinants of customer acceptance of multi-service network: An implication for IP-based technologies", Inform Manage 46(1), 16-22, 2009. https://doi.org/10.1016/j.im.2008.05.004

[20] Dalcher, I., Shine, J., "Extending the new Technology Acceptance Model to measure the end user information systems satisfaction in a mandatory environment: A bank's treasury", Technol Anal Strateg, 15(4), 441-455, 2003.

https://doi-org.ezproxy.ub.gu.se/10.1080/095373203000136033

[21] Krishnaraju, V., Mathew, S., Sugumaran, K., "Web personalization for user acceptance of technology: An empirical investigation of E-government services", Inform Syst Front, 18(3), 579-595, 2016. https://doiorg.ezproxy.ub.gu.se/10.1007/s10796-015-9550-9

[22] Lee, J-H., Song, C-H., "Effects of trust and perceived risk on user acceptance of a new technology service", Soc Behav Personal, 41(4), 587-598, 2013. http://dx.doi.org/10.2224/sbp.2013.41.4.587

[23] Teo, T., "Modelling technology acceptance in education: A study of preservice teachers", Comput Educ, 52(2), 302-312, 2009. https://doi.org/10.1016/j.compedu.2008.08.006

[24] Šumak, B., Heričko, M., Pušnik, M., "A meta-analysis of e-learning technology acceptance: The role of user types and e-learning technology types", Comput Hum Behav, 27(6), 2067-2077, 2011. https://doi.org/10.1016/j.chb.2011.08.005

[25] Strudwick, G., McGillis Hall, L., "Nurse acceptance of electronic health record technology: A literature review”, J Res Nurs, 20(7), 596-607, 2015. https://doi-org.ezproxy.ub.gu.se/10.1177/1744987115615658

[26] Pai, F-Y., Huang, K-I., "Applying the Technology Acceptance Model to the introduction of healthcare information systems", Technol Forecast Soc, 78(4), 650-660, 2011. https://doi.org/10.1016/j.techfore.2010.11.007

[27] Aggelidis, V. P., Chatzoglou, P. D.,"Using a modified technology acceptance model in hospitals", Int J Med Inform, 78(2), 115-126, 2009. https://doi.org/10.1016/j.ijmedinf.2008.06.006

[28] De Graaf, M., Ben Allouch, S., Van Dijk, J., "A phased framework for longterm user acceptance of interactive technology in domestic environments", New Media Soc, 1-22, $2017 . \quad$ https://doiorg.ezproxy.ub.gu.se/10.1177/1461444817727264

[29] Wagner, G. D., Flannery, D. D., "A quantitative study of factors affecting learner acceptance of a computer-based training support tool", J Eur Ind Training, 28(5), 383-399, $2004 . \quad \mathrm{https} / / / \mathrm{doi}-$ org.ezproxy.ub.gu.se/10.1108/03090590410533071

[30] Hasan, R. (2012). Systemic Functional Linguistics: Exploring Choice (Vol. 9781107036963). Cambridge University Press.

[31] Popova, O., Popov, B., Karandey, V. \& Evseeva, M. (2015). Intelligence amplification via language of choice description as a mathematical object (Binary Tree of Question-answer System). Procedia - Social and Behavioral Sciences, 214, 897-905.

[32] Hasan, R., \& Martin, J. (1989). Language development: Learning language, learning culture (Advances in discourse processes, 27). Norwood, N.J.: Ablex.

[33] Martin, J. R., "Meaning matters: A short history of systemic functional linguistics", WORD, 62:1, 35-58, 2016. https://doiorg.ezproxy.ub.gu.se/10.1080/00437956.2016.1141939

[34] Halliday, M. A. K. Language as social semiotic: The social interpretation of language and meaning, London: Edward Arnold, 1978.

[35] Whorf, B. L. Language, thought and reality: Selected writings, Cambridge, MA: MIT Press, 1956.

[36] Butler, C., "An ontological approach to the representational lexicon in Functional Discourse Grammar", Lang Sci, 34(5), 619-634, 2012. https://doi.org/10.1016/j.langsci.2012.02.004

[37] Bartlett, T. "I'll manage the context": context, environment and the potential for institutional change. In, L. Fontaine, T. Bartlett and G. O'Grady (eds.), Systemic functional linguistics: Exploring choice, Cambridge University Press, p. 342-364, 2013.

[38] Bourdieu, P., Language and symbolic power, Cambridge: Polity Press, 1991.

[39] Wilber, K., A theory of everything, Boston: Shambhala, 2000.

[40] Butler, C., "Criteria of adequacy in functional linguistics", Folia Linguist, 43(1), 1-66, 2009.

https://doi-org.ezproxy.ub.gu.se/10.1515/FLIN.2009.001 